\title{
Dentin Permeability: The Basis for Understanding Pulp Reactions and Adhesive Technology
}

\author{
Ivar A. Mjör \\ University of Florida College of Dentistry, Gainesville, FL, USA
}

\begin{abstract}
Permeability involves the passage of fluids, ions, molecules, particulate matter and bacteria into and through a substance or tissue under different and varying conditions. The permeability of the dentin is essential to support the physiology and reaction patterns of the pulp-dentin organ. Nutrients and impulses are transported from the pulp via the odontoblast process and the contents of its tubules maintain the dentin as a vital tissue. However, the main interest of this paper focuses on penetration from the outside towards the pulp rather than from the pulp towards the outside. The present overview centers on the dentinal tubules; how they are formed and how they change as a result of normal and abnormal function, age, and pathological processes and the effect of these processes on the permeability of dentin. Particular attention is focused on the patency of the dentinal tubules. This overview is largely based on the author's own research, clinical insights and active participation in continuing dental education over the last 50 years. It is not a review of the literature related to the permeability of dentin. Rather it presents interpretation of results related to the permeability of dentin based on experience and opinions acquired over a lifetime in dental research.
\end{abstract}

Key Words: dentinal tubules, age changes, caries, smear plugs, adhesive dentistry.

\section{INTRODUCTION}

This review will deal with variations in the structure of the dentin and its effect on the permeability of the dentin. Dentin may have open tubules as in newly erupted teeth and in other situations and in specific locations, it may have tubules that are partly or completely obturated or occluded by mineralized deposits. Some parts of root dentin have relatively few tubules. These differences will affect the permeability of the tissue, including the transfer of noxious agents, and consequently variation in dentin permeability will have an effect on pulp reactions. Furthermore, occluded tubules cannot be penetrated by restorative materials. Thus, the permeability of dentin becomes an integral part of modern restorative dentistry where adhesive technology plays a central role. The patency of dentinal tubules will therefore be a focal theme in this review.

\section{Pulp-Dentin Complex}

The outer periphery of the dentin is greater at the periphery than at the pulp-dentin border, especially in the crown part of the tooth. Since the dentinal tubules are cylindrical in shape and extend through the entire width of the dentin, the density of tubules is greater near the pulp than peripherally. In addition the branching of the tubules varies in different parts of teeth resulting in distinct differences in the structure of dentin (1). These differences are likely to affect the permeability of the tissue. In addition, post eruptive changes take place in dentin as a result of age and functional stimuli as well as by pathological processes like caries throughout the life of the tooth. In this review emphasis will be placed on the structural changes that affect the permeability of the tissue.

The dental pulp is one of a few tissues in the body that exhibit a normal relatively high interstitial tissue fluid pressure. This means that a pressure gradient exists in dentinal tubules under normal conditions. Anything trying to penetrate through the dentin towards the pulp must do so against a pressure gradient while anything penetrating from the pulp will be facilitated by the same pressure gradient provided the pulp is vital. This pressure gradient can be imitated in vitro by using an experimental design to compensate for the pressure 
gradient in the pulp in extracted teeth. Numerous factors will affect the permeability of dentin in vivo (2).

\section{Dentin Permeability}

Permeability refers to the state or quality of being open to passage, especially fluids, ions, bacteria, and minute particles. In physics it refers to the ease of passage and/or the rate of diffusion through a body or tissue under standard conditions. Many factors affect this passage, including the area exposed, the structure and chemistry of the involved tissue, the thickness of the tissue and the pressure exerted on the process. Detailed knowledge of the dentin and its reaction potentials is therefore essential in order to understand the tissue's permeability.

Particle size is also recognized as important in the demonstration of dentin permeability as well as any chemical interaction between the dentin and the penetrating agent. The penetration of bacteria associated with caries and the demonstration of leakage at tooth/restoration interfaces and into the dentin has been studied. The use of isotopes which allowed autoradiograms to be prepared by placing ground sections on X-ray film facilitated the visualization of the penetration of the radioactive isotope (3). The health hazard associated with the use of radioactive materials lead to a reactivation of the dye penetration studies which had been in use since the early days of dental research. Dye penetration allows visualization of the permeability, although care must be taken with dyes that could act as redox indicators and be colorless at a certain $\mathrm{pH}$ ranges.

\section{Dentin - Its Formation, Structure, Age Changes and Reaction Patterns (4)}

\section{Dentinogenesis}

Dentinogenesis starts in the cuspal/incisal area of the amelodentinal junction and dentin constitutes the main tissue of teeth. It is structurally characterized by a mineralized matrix that is mainly hydroxyapatite and it comprises about $70 \%$ of the tissue by weight and $50 \%$ by volume. Collagen is the main organic component of the dentin. It includes about $93 \%$ of the organic material and is formed during dentinogenesis. Citric acid is present and additional minor organic components include glycosaminoclycans, lipids and various protein components.
Formation of the crown portion of dentin starts at the bell stage of the tooth buds while they are embedded in the jaws. The mineralized dentin matrix is penetrated by tubules that extend through the full width of the tissue (Figs. 1a-d and 2a-d). The main bulk of the dentin is evenly mineralized but with distinct differences in degree of mineralization in specific locations. In coronal dentin the mantel dentin adjacent to the enamel, interglobular areas which are regularly found in the dentin close to the enamel and cementum and a zone close to the pulp are less mineralized than the bulk of the dentin (Figs. 1a-c). The peritubular dentin becomes highly mineralized in the bulk of coronal dentin during dentinogenesis (Figs. 1c-d and $2 \mathrm{a}-\mathrm{c}$ ) and it contains a scanty organic matrix (Fig. $2 d)$. After the tooth crown is formed, the secondary dentin formed while the tooth is embedded in the jaw and later in life has tubules without a highly mineralized peritubular dentin as primary structures (Figs. 1c and 3a-b).

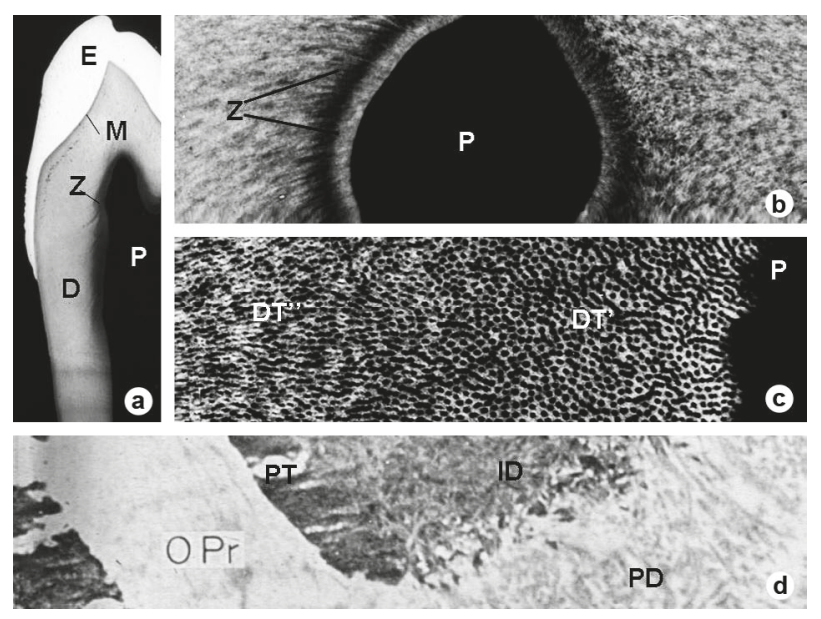

Figure 1. Panel of images from undemineralized sections of human premolars. Figs. 1a-c are microradiographs from newly erupted teeth. Fig. 1a is from a section through the central part of a premolar and Fig. $1 \mathrm{~b}$ is a section through a pulp horn. Fig. $1 \mathrm{c}$ shows cross sectioned dentinal tubules from the predentin $(\mathrm{P})$ extending peripherally. The dentin near the pulp and predentin in the coronal part of teeth is unevenly mineralized giving a zonated (Z) appearance. The dentin near the predentin has no highly mineralized peritubular dentin (DT') while further peripherally all tubules have highly mineralized peritubular dentin (DT"). E: enamel; D: dentin; M: mantle dentin; P: pulp and predentin. Fig. $1 \mathrm{~d}$ is a transmission electron micrograph of an unerupted tooth during dentinogenesis. Note that the peritubular dentin develops as a highly mineralized structure (PT) at the same time as the intertubular matrix (ID). PD: predentin; OPr: odontoblast process. Source: Takuma (12). 
The tubules have a diameter of $2-3 \mu \mathrm{m}$ at the time they are formed. In the main bulk of the dentin the tubule is $1-2 \mu \mathrm{m}$ in diameter (Figs. 2a-c). They house the odontoblast process, which remains after the matrix has formed as an extension of the odontoblast into the dentin. The presence of cytoplasm in the odontoblast process is a continuation of that in the odontoblast itself, but it is limited in extent to the most pulpal part of the process in newly erupted teeth (Figs. 3a-b). The odontoblast process contains few organelles associated with matrix formation like endoplasmic reticulum, Golgi apparatus and mitochondria. In the main part of the dentin, the tubules contain remnants of the odontoblast process and tissue fluids and they may be obturated by growth of the peritubular dentin (Figs. 4a-b) as an age change or by mineral deposits. Such deposits reduce the size of the lumen and they may occur under a number of conditions, e.g., as a result of caries, restorative procedures, or exposure to the oral environment. Complete occlusion of the tubules by mineral depositions may take place and
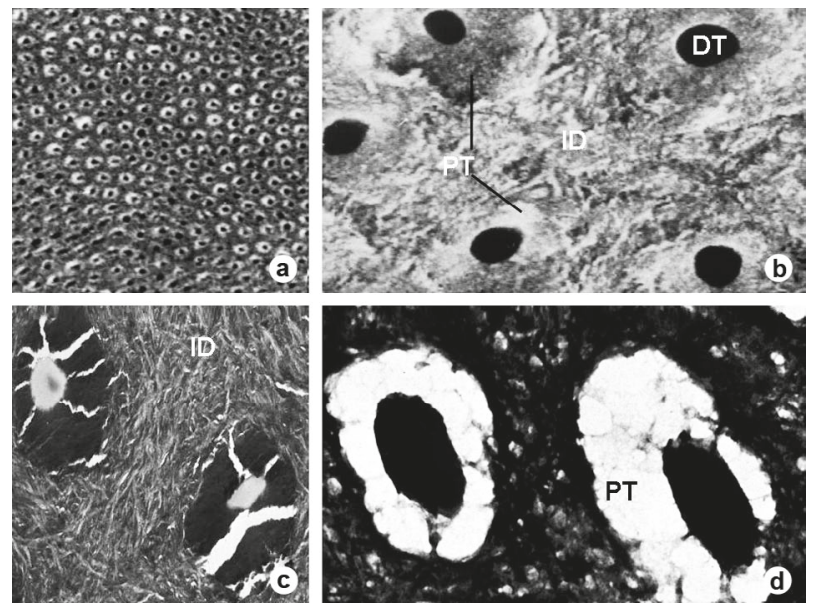

Figure 2. Panel of images from cross-sectioned dentinal tubules from human coronal dentin. Fig. 2a is from a microradiograph of a ground section showing all tubules lined by a highly mineralized (white) peritubular dentin. Fig. $2 b$ is a scanning electron micrograph of a fractured tooth showing dentinal tubules (DT) with peritubular dentin (PT). ID: intertubular dentin. Fig. $2 \mathrm{c}$ is a transmission electron micrograph showing two cross sectioned dentinal tubules with highly mineralized peritubular dentin (black) which cracks easily during sectioning. Fig $2 \mathrm{~d}$ is a transmission electron micrograph showing two cross- sectioned dentinal tubules. The section was demineralized on the grid while the organic component was stained by phosphotungstic acid supporting the section. Note the scanty organic matrix of the peritubular dentin (PT). then the dentin becomes impermeable and transparent.

The two mineralized components of the dentin, the peritubular and intertubular matrices, mineralize at
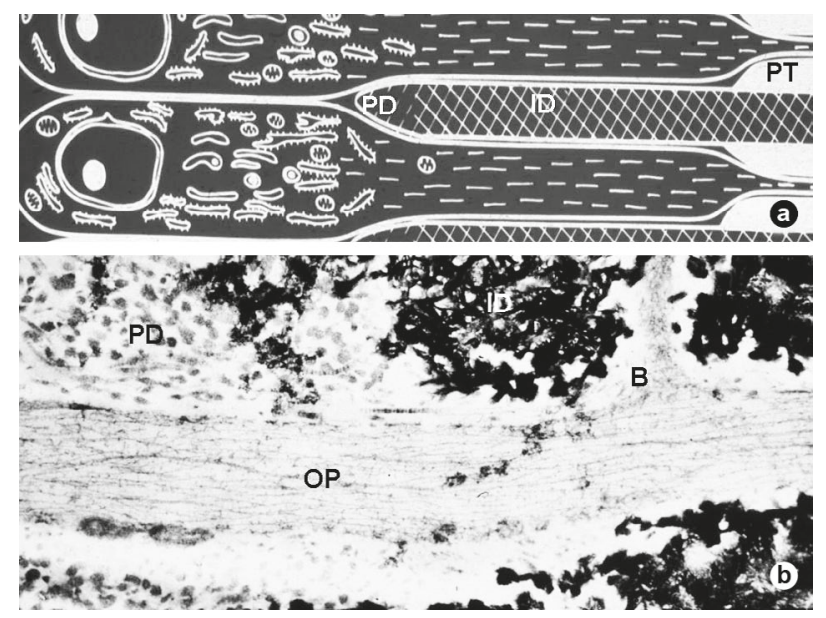

Figure 3. The ultrastructure of odontoblasts. 3a is a diagram of two odontoblasts with numerous organelles in the body of the cell with only microtubules in the odontoblast processes. PD: predentin; ID: intertubular dentin; PT: highly mineralized peritubular dentin. Fig. $3 \mathrm{~b}$ is from a transmission electron micrograph of an undemineralized specimen showing an odontoblast process (OP) with a side branch (B). PD: predentin; ID: intertubular dentin.
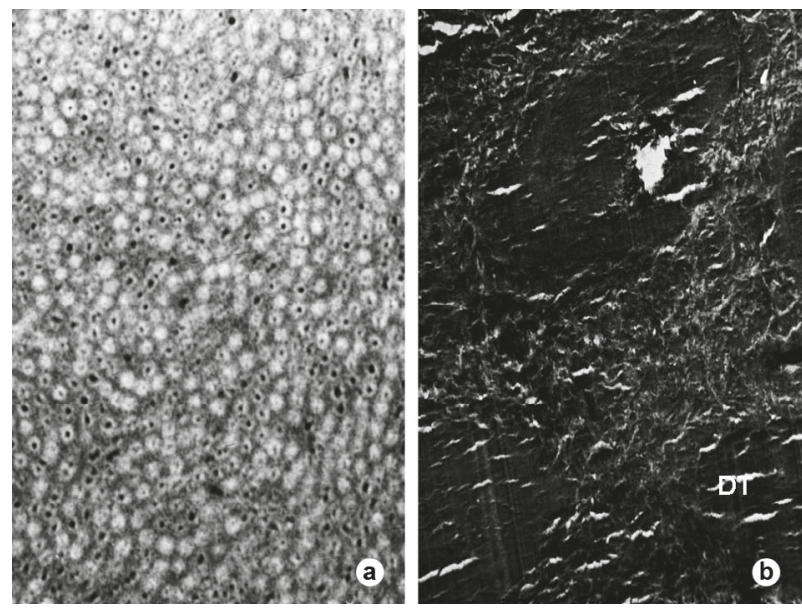

Figure 4. Images from undemineralized sections of dentin from teeth of old individuals. In Fig. 4a, most of the cross-sectioned dentinal tubules are obturated by growth of the peritubular dentin (white) when viewed in a microradiograph. Fig. $4 \mathrm{~b}$ is a transmission electron micrograph in which the occluded (DT) and partly occluded tubules appear black. 
the same time during dentinogenesis, i.e., they are both primary developmental structures in the main portion of the dentin. The peritubular matrix mineralizes to a much greater extent than the intertubular matrix (Figs. 1c-d and 2a-c). It is likely that the highly mineralized peritubular dentin markedly reduces any transport of fluids, ions or molecules from the tubules to the intertubular matrix. The potentials for changes in the intertubular matrix after the dentin is formed are therefore limited. Any organic matrix formed within the tubules after the tooth is erupted will depend on the diffusion of the components through the remnants of the odontoblast process and the intratubular tissue fluid, including that in the periodontoblastic space.

\section{The Periodontoblastic Space}

The periodontoblastic space is a liquid filled space located between the odontoblast process or its remnants and the wall of the tubule (Figs. 3a-b). This 'space' is the likely route of transport of components which are involved in post eruptive growth of the highly mineralized peritubular dentin. It will increase in size as an age change, and it may also form as a secondary structure in the tubules in the most pulpal part of the dentin. These activities are considered to be under the control of the odontoblasts and as such they represent a physiological process. Growth of the peritubular dentin may result in complete obturation of the tubules which is a characteristic age change of dentin (Figs. 4a-b), and it may be accelerated as a result of external influences.

\section{Smear Layer}

The smear layer covers cavity preparations. It develops as a result of grinding of enamel and dentin or preparation of a root canal. It is a mechanical process whereby small ground particles combined with saliva or any liquid present are forced or smeared onto the surface and often into the tubules covering the prepared surface. The smear layer and the smear plugs in the opening of the tubules will reduce the permeability of the dentin (Figs. 5a-b). Washing the dentin with a water spray or irrigating a root canal will not remove the smear layer and the smear plugs, but they are easily and quickly removed by acid etching (Figs. $5 \mathrm{c}-\mathrm{d}$ ). Chelating agents at a neutral $\mathrm{pH}$ have also been used to remove the smear layer, e.g., removal of the smear layer in root canals, but the removal of the smear layer is difficult to control in root canals.

The long-term effect of the smear layer and smear plugs is dependant on the $\mathrm{pH}$ of the restorative material or cement placed in direct contact with the dentin. Polishing the prepared surface with pumice in a rubber cup will remove the smear layer and leave many smear plugs in place (Fig. 5a) while acid etching of a dentin surface will remove the smear layer and the smear plugs (Figs. 5c-d) leaving a mesh of collagen on the prepared surface (Fig. 5d) provided careful demineralization takes place and the surface remains wet. This mesh of collagen when infiltrated with resin monomer and polymerized, forms the so-called 'hybrid layer'. This layer is an essential part of adhesive dentistry.

\section{Types of Dentin}

In this review, the terms primary dentin, second-
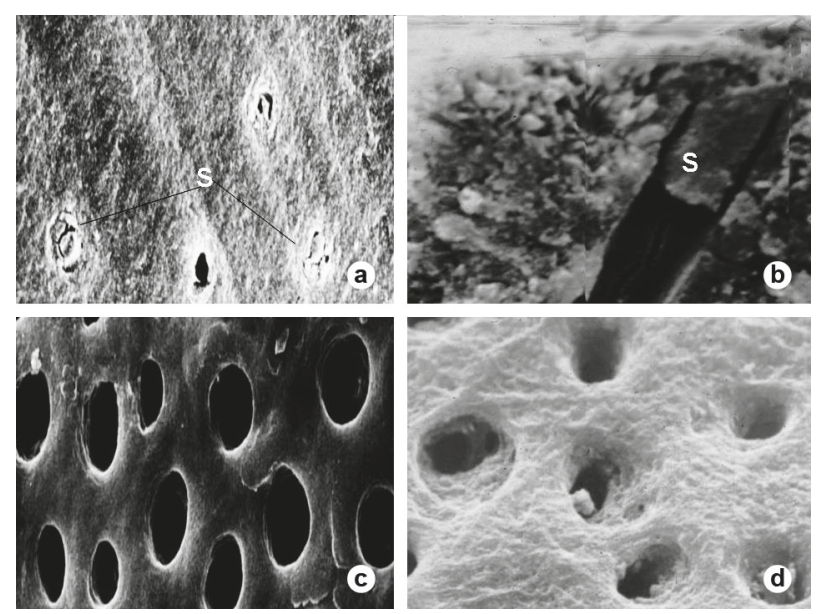

Figure 5. Panel of scanning electron micrographs showing cross-sectioned $(5 \mathrm{a}, \mathrm{c}, \mathrm{d})$ and longitudinal $(5 \mathrm{~b})$ views of dentinal tubules. Fig. 5a is from a surface of a crown preparation that was polished using a slurry of pumice in a rubber cup and washed with a water spray. Note a smear plug (S) in two of the dentinal tubules. 5b shows a smear plug (S) in a dentinal tubule after cavity preparation. Figs $5 \mathrm{c}$-d shows a prepared dentin surface acid etched with $35 \%$ phosphoric acid for $60 \mathrm{~s}$, washed with a water spray and dried with an air syringe (Fig. 5c). After acid etching of the prepared dentin for $20 \mathrm{~s}$ and gentle dying to remove excess water, a collagen mesh remains (5d) and it may be impregnated with a low-viscosity resin bonding monomer, which is cured leaving an oxygen inhibited surface layer for bonding to the resin-based restorative material. The network of collagen fibers on the prepared surface $(5-10-\mu \mathrm{m}$-thick) becomes the hybrid layer after it is impregnated by resin in the completed restoration. 
ary dentin and tertiary dentin will be used. Primary dentin is formed during odontogenesis. Secondary dentin starts forming while the tooth is still embedded in the jaws and it continues after the tooth is erupted. Both primary and secondary dentin formation are under control of the odontoblasts that form the main bulk of dentin. The tubules in the two types of dentin form a continuum. Tertiary dentin forms in response to external stimuli and it is limited in extent to the external stimulus (Figs. $6 \mathrm{a}-\mathrm{c})$. It is formed by secondary odontoblasts that have differentiated from pulp cells after the primary odontoblasts have been destroyed (Fig. 7a).

\section{Physiological Secondary Dentin}

After the enamel of the crown is fully formed, the crowns of permanent teeth remain in the jaw for 2-3 years prior to tooth eruption. Dentinogenesis continues at a slow rate during this time and the dentin is initially less mineralized than in the bulk of the dentin and only the intertubular matrix mineralizes (Fig. 1c). Any subsequent dentin formed is referred to as secondary dentin. It continues to be formed after the tooth has erupted and the degree of mineralization of the intertubular matrix assumes a higher degree of mineralization similar to that in the main part of the dentin, giving the

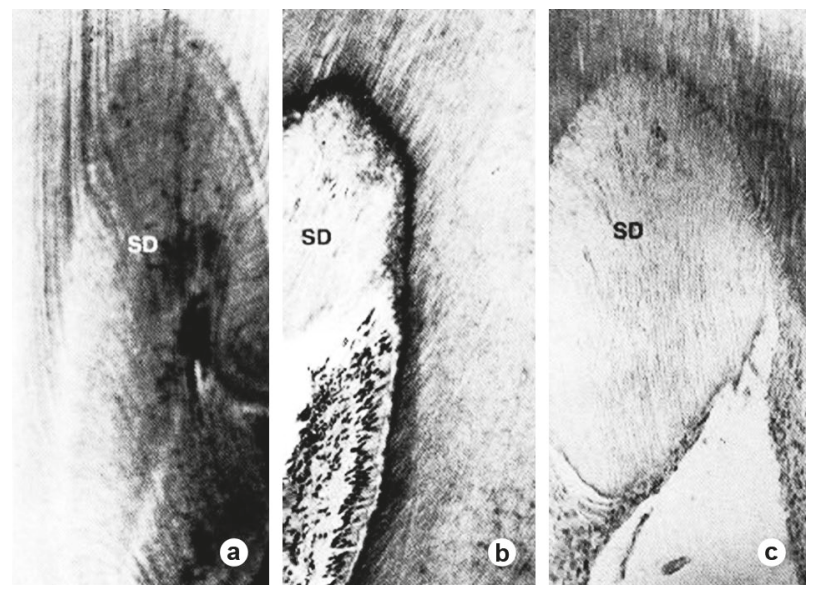

Figure 6. Secondary dentin (SD) in the cuspal area of sections of worn teeth. Fig. 6a is a microradiograph of a ground section and Figures $6 \mathrm{~b}-\mathrm{c}$ are from stained demineralized sections. Note the irregular structure of the first formed secondary dentin and it may have cellular inclusions (Fig. 6b) which is characteristic for tertiary dentin. In some areas the dentinal tubules continue from primary to secondary dentin. coronal dentin a zonate microradiographic appearance (Figs. 1a-b). This particular mineralization pattern is a characteristic feature of coronal dentin and it is not found in root dentin. It is likely that the less mineralized zone in coronal dentin, i.e., the dark zone seen microradiographically of ground sections of erupted teeth (Fig. 1b), remains hypomineralized throughout the life of the tooth. It delineates the transition from primary to physiological secondary dentin. The dentin pulpal to this hypomineralized zone represents the first formed secondary dentin, and it has tubules which lack highly mineralized peritubular dentin. Root formation starts at the same time. Whenever highly mineralized peritubular dentin can be identified in this dentin, they are secondary structures.

Secondary dentin formation continues at a slow rate during the life of the tooth both in the crown and in the root. It should be differentiated from localized irregular secondary dentin, which is a type of tertiary dentin formed in response to post eruptive external stimuli.
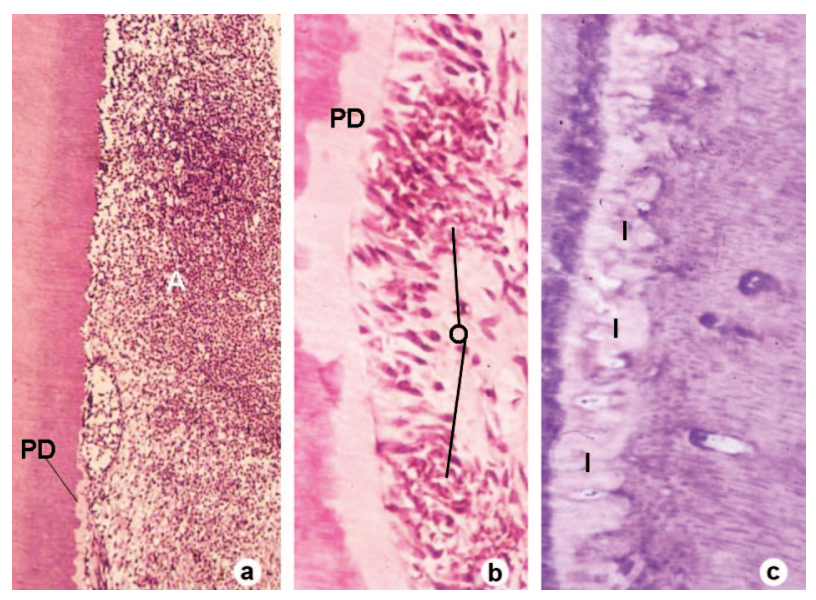

Fig. 7. Panel of images of hematoxylin and eosin stained sections of demineralized monkey teeth that were part of an experimental series. Fig. 7a shows an experimentally induced, localized abscess (A) which has destroyed the odontoblast layer. The predentin has mineralized in the affected area. PD: predentin adjacent to the localized abscess. Fig. 7b shows a partly healed abscess from an experimental series. Note cellular infiltration in the odontoblast region (O). PD: predentin. Fig. 7c shows the interface dentin (I) which was formed as the abscess similar to that shown in Fig. 7a was allowed to heal. Note lack of dentinal tubules in the interface dentin and the presence of cellular inclusions in the predentin and dentin. As the healing progressed, the tertiary dentin assumed a structure similar to that of secondary dentin with tubules. 


\section{Tertiary Dentin}

Tertiary dentin is the tissue formed as a response to localized, strong external stimuli in functional teeth, e.g., localized severe inflammation (Fig. 7a). It is formed by secondary odontoblasts differentiated from undifferentiated cells in the pulp. Such cells are abundant in newly erupted teeth, but they are reduced in numbers as an age change in the pulp. If some odontoblasts survive the influence of the external stimulus, they may continue to form secondary dentin (Figs. 6a-c).

Tertiary dentin is irregular in structure and localized to the affected dentinal tubules. The first part of it is formed at the time of the differentiation of secondary odontoblasts. Fibroblasts in the pulp may form part of the matrix at this stage and cellular inclusions in the matrix may occur (Figs. 6b and 7c). This matrix lacks dentinal tubules (Fig. 7c). Continued tertiary dentin formation results in a tissue with tubules that is similar in structure to that in primary and secondary dentin (Fig. 7c).

It is important when considering the permeability of dentin to realize that the tertiary dentin is not formed by the same odontoblasts as those forming the primary dentin and its continuation as secondary dentin. This atubular mineralized tissue may appropriately be referred to as 'interface dentin'. Since the tubules from the primary/secondary dentin do not continue into the tertiary dentin, it will have a marked effect on the permeability of the dentin by acting as a barrier to any penetration

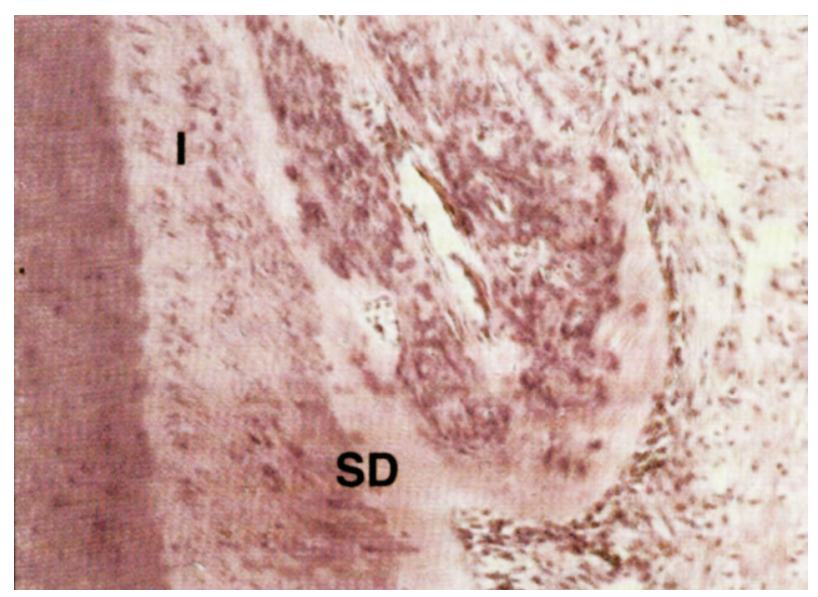

Figure 8. Hematoxylin and eosin stained section of a demineralized tooth which had been extracted and the immediately replanted. Note the interface dentin (I) and the secondary dentin which has formed three months after replantation. from the peripheral dentin. In that way a classical 'dead tract' in the dentin has been formed (5).

A special form of tertiary dentin may be found under extreme conditions, e.g. after replantation of extracted newly erupted vital teeth. This tissue response may result in the formation of large masses of irregular, bone-like tissue in the pulp (Fig. 8), and it is not considered relevant in a discussion of dentin permeability. However, all or most of the coronal dentin then becomes a 'dead tract'. Thus no vital reactions can occur in the affected dentin.

\section{Root Dentin}

The root portion of the dentin is formed during
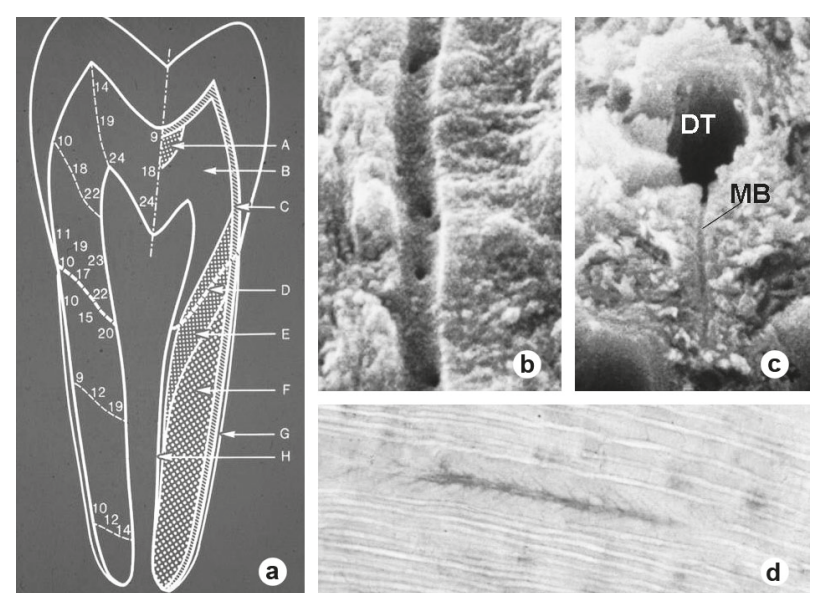

Figure 9. The different types of branching of dentinal tuules. Fig. 9a shows a diagram tooth illustrating on the left half the number of dentinal tubules in different locations per $100 \mu \mathrm{m}$ of dentin counted across the long axis of the tubules at three different levels in dentin on demineralized, stained sections of teeth. The right half of the diagram identifies eight areas of dentin that can be identified on the basis of type of branching and density of tubules: A, area under occlusal fissure; B: main part of coronal dentin and the most pulpal part of the root dentin; C: outer $250 \mu \mathrm{m}$ of dentin in crown and root; D: the dotted line corresponds to the dentinal tubules at the cementoenamel junction; $\mathrm{E}$ : transition zone on each side of D; F: main part of root dentin; G: cementum; H: predentin. Source: Mjör and Nordahl (1). Figs. 9b-c are scanning electron micrographs of fractured teeth showing a longitudinal view of a dentinal tubule with many openings for fine branches (Fig. 9b). Fig. 9c shows a microtubule (MB) leaving a dentinal tubule (DT). Fig. 9d is from a hematoxylin and eosin stained section of a demineralized tooth showing an unusual branching of a dentinal tubule in root dentin. Note that the branches emerge from the tubule at a $45^{\circ}$ angle. 
tooth eruption. The dentinal tubules are generally further apart in root dentin than in coronal dentin and they are characterized by an abundance of fine branches that fork off at about a $45^{\circ}$ to the tubule. A gradual intratubular mineralization of root dentin occurs as an age change and it may result in complete obturation of the dentinal tubules. Roots where the tubules have mineralized become transparent. Some parts of the root dentin have few tubules (6) and that will affect the permeability of the dentin.

\section{Branches of the Dentinal Tubules}

Different types of branches of dentinal tubules are found (Figs. 9a-d and 10a-d) and they can be differentiated on the basis of size, location and direction of the branches in relation to the main tubule (1). Major branches of the tubules are found peripherally up to
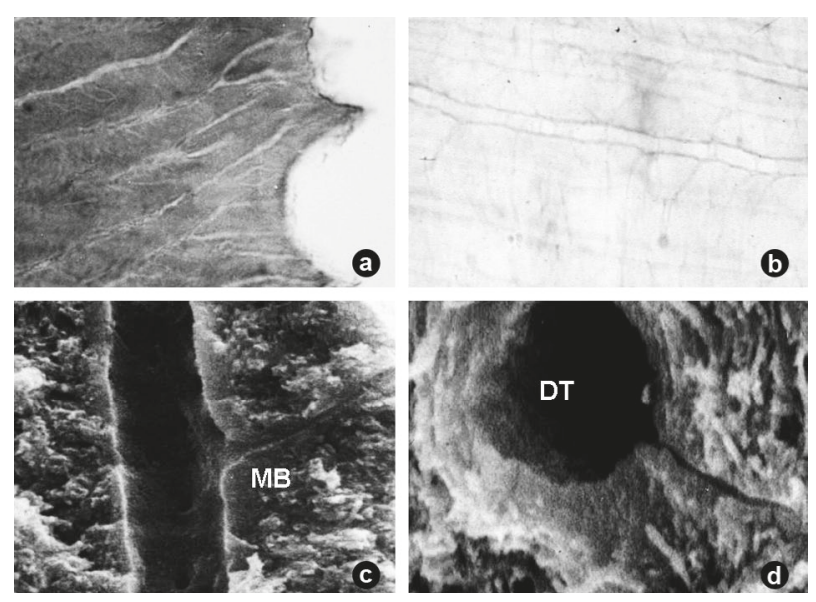

Figure 10. Different types of branching of dentinal tubules. Fig.10a is from a section of a Masson stained, demineralized tooth showing typical delta branches in the mantle dentin. Fig. 10b is from a hematoxylin and eosin stained section of a demineralized tooth showing fine branches of the tubules in root dentin. Most discernable branches extend from the tubules at a $45^{\circ}$ angle. Fig. 10c is a scanning electron micrograph of a fractured tooth showing a longitudinal view of a dentinal tubule with many fined branches leaving the tubule. One fine branch is sectioned longitudinally and the peritubular dentin extends along the branch. Note that the fine branch extends from the tubule at a $45^{\circ}$ angle which is typical for branching of tubules in root dentin. Fig. 10d is a scanning electron micrograph of a fractured tooth showing a dentinal tubule (DT) with peritubular dentin lining the tubule and extending along the microbranch. Note that the microbranch leaves the tubule at a $90^{\circ}$ angle. approximately $250 \mu \mathrm{m}$ from the outer surface in both coronal and root dentin. These branches form a delta and the diameter of the tubules ranges from 0.5 to $1.0 \mu \mathrm{m}$ (Fig. 10a). Fine branches are typical of root dentin and in any location where the number of tubules is low. They vary in diameter from 0.3 to $0.7 \mu \mathrm{m}$ and they branch off from the main tubule at about a $45^{\circ}$ angle (Figs. 10b-c). Microbranches can be found anywhere in dentin. They are up to about $0.1 \mu \mathrm{m}$ in diameter and branch off from the tubule at a $90^{\circ}$ angle (Figs. $9 \mathrm{c}$ and $10 \mathrm{~d}$ ).

Studying the branching of dentinal tubules leaves the impression that there is a relationship between tubule density and the presence of branches from the tubules, but an exact relationship has not been established. Since the branches and the tubules are likely pathways of fluids, ions and molecules such a relationship is to be expected in order to maintain the physiology and reactivity of the
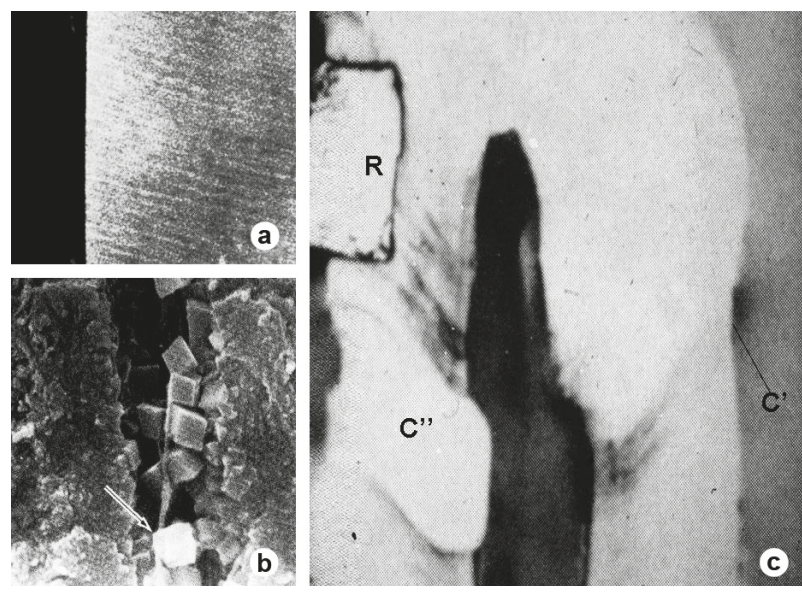

Figure 11. Panel of images from dentin that has been exposed to the oral environment without plaque formation on its surface. Fig. 11a shows a microradiograph of a dentin surface with increased mineralization after exposure to the oral environment. Fig. $11 \mathrm{~b}$ is a 'dentin biopsy' of the surface layer with higher mineral content examined in a scanning electron microscope showing that mineral salts have precipitated in the tubules. Source: Yoshiyama et al. (10). Fig. $11 \mathrm{c}$ is a bisected human canine that was placed in an isotope solution after extraction and an autoradiograph prepared. A small active and sensitive caries lesion (C') was present on the lingual surface at the amelocementum junction. The dentin subjacent to the lesion was permeable to the isotope and it penetrated into the pulp. An arrested Class V lesion (C") was present on the buccal surface of the tooth and it was impermeable to the isotope. Note that the Class V amalgam restoration (R) was leaking and the isotope penetrated through the dentin into the pulp. Source Barber and Massler (9). 
dentin. The periphery of at least some of the branches has a lining that is similar to the highly mineralized peritubular dentin (Figs. 9c and 10c-d). Some tubules in root dentin have unusual branching (Fig. 9d).

\section{The Number of Dentinal Tubules}

The number of dentinal tubules per $\mathrm{mm}^{2}$ varies markedly depending on the location within the dentin. The normal range in tubule density per $\mathrm{mm}^{2}$ is from about 5,000 to 90,000 . Due to the difference in the surface area of the dentin at the amelo- and cemento-dentinal junction and that at the predentin border in the fully formed and functional teeth, the tubules will be more densely packed on the pulpal aspect of the dentin than peripherally. The most extreme crowding of the tubules is found at the pulpal aspect subjacent to occlusal fissures, incisal edges and cusp tips. The lowest density of tubules is found peripherally and it does not vary much in the different parts of the tooth.

\section{Physical Occlusion of Tubules}

The presence of intratubular crystalline material of different morphologies has been demonstrated in hypermineralized areas of dentin. These deposits lead to obturation of dentinal tubules. They have been observed

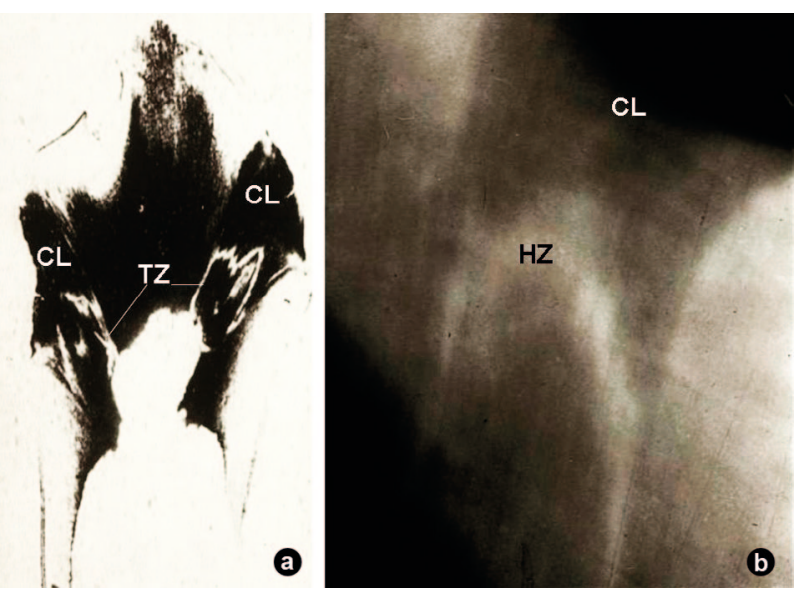

Figure 12. Images ( $a$ and $b$ ) illustrating the reactive zone in dentin subjacent to caries lesions (CL). In ground sections [Source Bergman (11)] this reaction zone in dentin appears transparent (TZ) in ground sections and in microradiographs it shows up as a hypermineralized area (HZ). in dentin exposed to the oral environment under self cleansing conditions (Figs. 11a-b), associated with caries (Fig. 12a-b), and following the use of some restorative materials (Figs. 13a-c and 14a-e). The effect of such occlusion of the tubules on the permeability of the dentin (Fig. 13c) is similar to that as a result of growth of the peritubular dentin. However, the mechanism involved in this intratubular mineralization is different. Growth of the peritubular dentin is a reaction under the control of odontoblasts, i.e., it is a vital reaction in the true sense of the term. Other deposition of minerals in the tubules is considered to be a physical process not dependent on cellular activity.

\section{Summary of Tissue Changes and Reaction Patterns In Dentin}

The structure and physiology of intact pulps in newly erupted teeth are well established and they

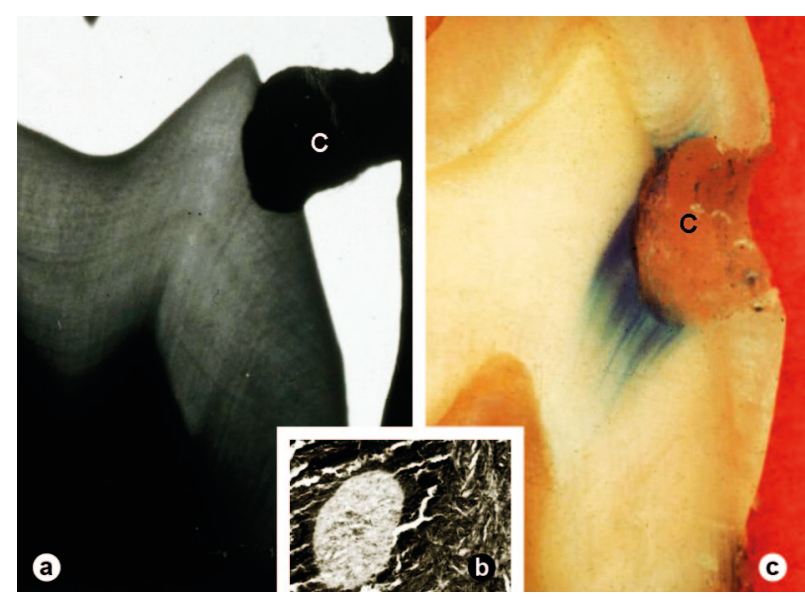

Figure 13. Panel of images from calcium hydroxide covered dentin in newly erupted teeth. Fig. 13a is from a microradiograph of a ground section through a restoration of an experimental cavity $(\mathrm{C})$ where calcium hydroxide was placed as a base and the rest of the cavity filled with amalgam. Note the increased mineralization of the dentin corresponding to the cavity. The increased mineralization of the tubules corresponding to the cavity is due to mineral crystals deposited in the tubules (Fig. 13b). Fig. 13c shows a bisected tooth that had been subjected to permeability studies using a methylene blue dye solution. The cavity $(\mathrm{C})$ had been restored with a calcium hydroxide base and amalgam. After extraction the restoration was carefully removed and the cavity exposed to the dye solution. Note that the dye only penetrated the outer part of the dentin while the pulpal part of the dentin was impermeable. 
are therefore considered suitable for experimental studies, but the availability of such human teeth is limited and ethical consideration also limits their use. Sometimes teeth to be extracted as part of orthodontic treatment of children become available. Such teeth have, therefore, been used extensively, but changes in orthodontic treatment modalities where tooth extraction is rare have limited their availability. Stricter emphasis on ethical considerations has also made such teeth less accessible. It must also be kept in mind that the results from experimental series using such teeth cannot be extrapolated to be valid for teeth that have been subjected to wear, clinical procedures, caries or restorative materials.

Teeth are subjected to several clinical conditions that may change their structure and physiology, e.g., age changes, caries, different types of wear (attrition, erosion, abrasion and abfraction), and restorative procedures, including the effect of different restorative materials. Changes take place intratubularly in the primary/secondary dentin continuum and at the pulpal
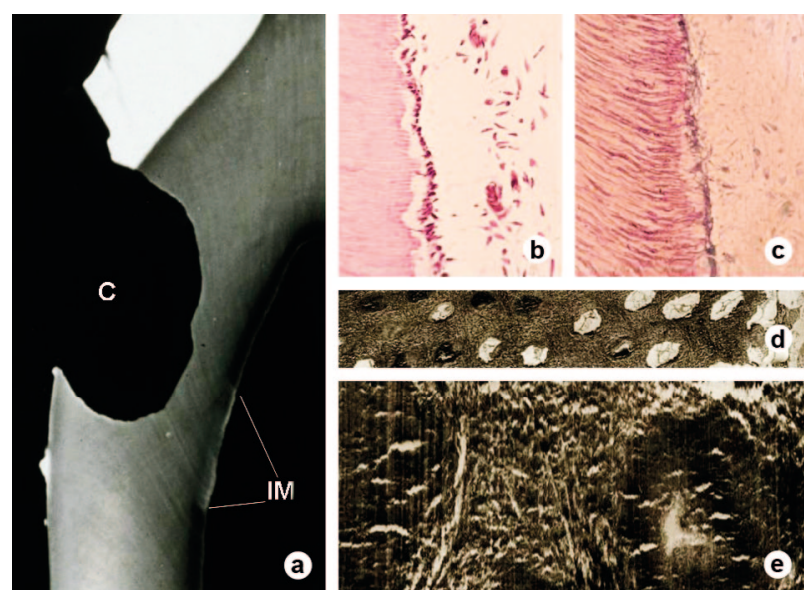

Figs 14. Panel of images from dentin that has been covered by a corticosteroid/antibiotic paste as a base and amalgam. Note the increased mineral content (IM) in the most pulpal part of the dentin subjacent to the restored cavity (C) (Fig. 14a). The rest of the dentin shows no change in mineralization. Hematoxylin and eosin (Fig. 14b) and toluidine blue stained (14c) demineralized sections from an area subjacent to corticosteroid/antibiotic paste (top half of the illustrations) applied similar to that in Fig.14a. A specimen dissected out from the area of increased mineral content (IM in Fig. 14a) examined in transmission electron microscope (Fig. 14d) shows that the tubules are fully or partially obturated by mineral deposits in the area that normally do not have highly mineralized peritubular dentin (Figs. 14d-e). end of the tubules by the formation of tertiary dentin. Both processes lead to a reduction in the permeability of the dentin.

The intratubular changes involved in the different reaction patterns in dentin as well as the formation of localized masses of tertiary or 'reactionary' dentin in response to a wide range of external factors are biological defense mechanisms of importance for the longevity of the teeth. Dentin which becomes hypermineralized post-eruptively due to deposition of mineral salts within the dentinal tubules will be less permeable and have reduced potentials for pulp reactions caused by noxious agents (Figs .11a-c). Thus, two different mechanisms of intratubular mineralization should be recognized: 1. A vital response under control of the odontoblasts, and 2. A physicochemical occlusion of the tubules by precipitation of mineral salts

Increased mineralization of the intertubular matrix after dentin is fully formed has not been shown; although it is feasible to occur when it is exposed to the oral environment under self-cleansing conditions (Fig. 11a).

The primary dentin may be demineralized in vivo and it will affect both inter- and intratubular tissue. Apart from the demineralization that occurs due to

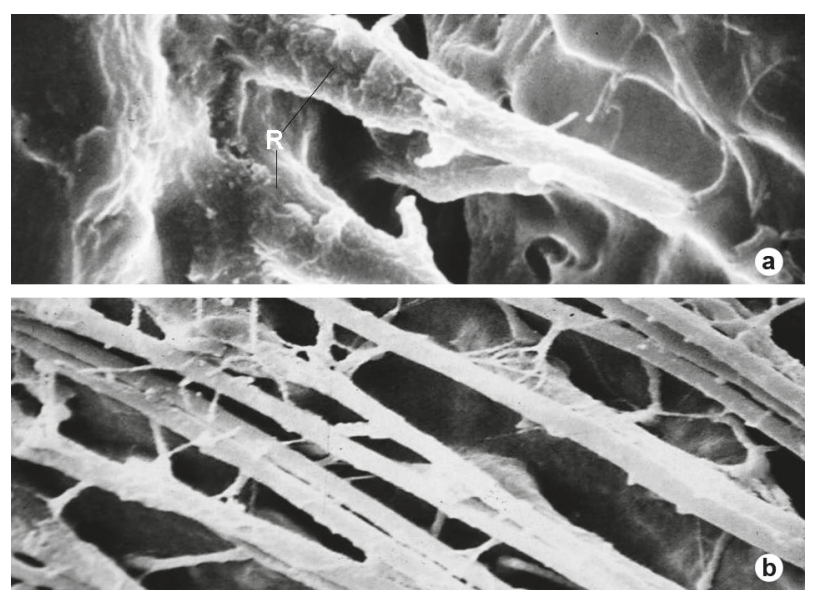

Figure 15. Scanning electron micrographs showing resin tags from restorations into dentinal tubules. The dentin was demineralized and the organic matrix digested away. Fig. 15a shows a coneshaped resin tag next to a restoration $(\mathrm{R})$. The cone-shape is a result of the limited demineralization of the highly mineralized peritubular dentin of the prepared surface as a result of the acid etching. Fig. $15 \mathrm{~b}$ shows resin extending into dentinal tubules and their fine branches. 
caries, intentional demineralization is also part of the bonding technique when using resin based restorative materials. The permeability of dentin will be enhanced by demineralization and it will facilitate the penetration of resin monomer into tubules (Figs. 15a-b). The highly mineralized peritubular dentin will demineralize quicker by the acid-etch procedure than the intertubular matrix making resin tags conical in shape (Fig. 15a). Demineralization will also expose a 5-10 $\mu \mathrm{m}$ thick mesh of intertubular collagen (Fig. 5d) to allow the formation of a hybrid layer which is essential for bonding resin based materials to dentin. By impregnation of the exposed collagen mesh with monomer to become the hybrid layer after polymerization it will reduce the permeability of the dentin. The monomer may also penetrate into the branches of dentinal tubules (Fig. 15b) which will enhance the retention of bonded resin restoration.

Teeth from animals may also be used, but the interpretation of the results is always hampered by possible differences in reactivity of animal teeth and human teeth. The structure of the dentin and its reaction patterns may be different from those in human teeth which limits their clinical application, although results from teeth of non-human primates are likely to be similar to those in human teeth. Ethical consideration must also apply to the use of animal teeth.

\section{Clinical Conditions that Affect Dentin Permeability}

The clinical conditions that have been reported to affect or be associated with dentin permeability are age changes, dentin hypersensitivity, different types of wear, biological reactions to restorative materials, dental caries and bonding to dentin.

The structural changes in the dentin under these conditions will be outlined with special reference to the predominant tissue changes as described above and how they affect the permeability of the dentin.

\section{Age Changes}

Age changes involve a gradual increase in the mineralization of the dentin. It occurs as a growth of the peritubular dentin which may result in occlusion of the tubules. This type of change was one of the first to be noticed by light microscopy because it altered the dentin's reflective and transmitted light properties in ground sections. It was described as 'transparent' dentin and it was associated with reduced permeability. The clinical implications of the age changes are that the degree of pulp reactions as a result of external noxious agents will be less in old than in newly erupted teeth due to the reduced permeability of the dentin. In this context clinicians should be reminded of the fact that studies on pulp reactions to restorative procedures have largely been performed on newly erupted, intact teeth which are maximally permeable.

\section{Dentin Hypersensitivity}

Dentin exposed to the oral environment may cause acute, sharp pain. The pain may be initiated by touching the dentin surface by an instrument, a finger nail or by the bristles of a tooth brush. Cold foods or drinks and sweets may result in pain of short duration. These reactions are usually referred to as hypersensitivity reactions and they must be differentiated from long lasting tooth ache which is pulpal pain. A special dentin biopsy technique has been developed, allowing dentin biopsies from sensitive and non-sensitive dentin to be studies. The results show that the non-sensitive dentin is hypermineralized and that the increased mineral content is due to occlusion of tubules by crystalline material. Hypersensitive dentin is permeable, while exposed nonsensitive dentin is impermeable (Fig. 11c).

\section{Different Types of Wear}

Slow wear of teeth during normal function, has a stimulating effect on post-eruptive changes in dentin resulting in enhanced mineralization. These changes can only be differentiated from age changes by their localization subjacent to wear facets (6). Functional wear leading to minor attrition usually occurs in incisal or cuspal areas where maximal crowding of the odontoblasts is normally found. Some odontoblasts will therefore be lost due to crowding and the localized increased formation of tertiary dentin will be irregular in structure (Figs. 6ac), but without the formation of interface dentin. This tertiary dentin will have variable structure, depending on the number of primary odontoblasts that remain.

Quick and excessive attrition or grinding of teeth will exacerbate the loss of enamel and dentin. The dentin changes will be similar to those following exposure of dentin to the oral environment and hypersensitivity reac- 
tion is a common clinical symptom because the tubules are not occluded by mineral deposits. If left open to the oral environment and the surface is kept free of plaque, mineral deposits in the tubules will reduce or eliminate the hypersensitivity (Fig. 11c).

Abrasion denotes wear caused by a specific agent, e.g., associated with vigorous tooth brushing with stiff brush bristles or by holding of instruments between teeth while performing specific routine tasks. Toothpastes also vary in the type and coarseness of the abrasive component. The abraded surface is often glossy and hard without clinical symptoms. The dentin changes are similar to those seen associated with attrition during normal function of the teeth.

Erosion of teeth represents a chemical degradation of the mineralized tissues of the tooth. The clinical consequence is hypersensitivity of the dentin and it continues as long as the acid effect continues due to a lack of obturation of the tubules. The hypersensitivity will be reduced when tertiary dentin forms on the tubules affected by the erosion and in that way it blocks off the tubules from the oral environment (Fig. 11c).

Abfraction is due to abnormal bending of teeth during function. It disturbs the enamel prisms over time leading to disruption and localized cracking of the enamel. It may result in exposing the dentin and the changes in the dentin will then be similar to those associated with abrasion.

\section{Biological Reactions to Restorative Materials}

Numerous studies of pulp and dentin reactions to restorative materials have been carried out, but these have usually been done on intact, newly erupted teeth. Teeth to be extracted for orthodontic reasons have been commonly used. This situation clearly limits the clinical significance of the results to be valid only for intact, newly erupted teeth. The reason for using newly erupted teeth is that their structure and physiology are well established. Thus, reactions to cavity preparation must first be established and that must be the basis for pulp studies to evaluate the effect of restorative materials. Techniques involving cavity preparations under abundant water spray have been used, and for the most common histopathological techniques used, the effect of cavity preparation per se can be controlled and is assumed to cause no changes. Using such a non-traumatic cavity preparation technique allows the study of restor- ative materials after restorations have been placed and remained in situ for variable observation periods.

The use of newly erupted teeth limits their clinical significance, because effects of age, wear and caries will not be part of the assessment. The effect of all these variables which affects all teeth treated clinically cannot be evaluated. However, some results from such studies will be outlined in order not only to show the effect on intact, newly erupted teeth, but also to outline potential effects that may occur under other situations.

Intratubular crystalline material has also been shown after calcium hydroxide mixed with water was applied to dentin in cavity preparations in intact, young teeth (Figs. 13a-c). Such depositions of crystals (Fig. 13b) result in an increased hardness and radiopacity of the dentin (Fig. 13a). The permeability is also reduced (Fig. 13d).

Similar depositions of crystals have not been reported following the application of other restorative materials. However, an intratubular matrix formation which results in localized hypermineralization of the affected dentin has been reported following the application of a corticosteroid-antibiotic paste on dentin in cavity preparations in newly erupted teeth (Figs. 14a-e). Localized intratubular matrix formations and mineralized deposits have been reported even in locations were highly mineralized peritubular dentin is normally not found (Fig. 14d). These tissue responses must be under the control of odontoblasts. This unique mineralization takes place within 30-40 days in human teeth and it results in an obturation of tubules (Figs. 14d-e) similar to that seen as an age change. It will affect the permeability of the affected dentin, but is permeability has not been tested in practice.

\section{Dental Caries}

It is important to clinically differentiate between acute and chronic/arrested caries. The clinical criteria for acute dentin caries are that the tissue is demineralized and soft, the color is yellow or yellow/orange and looks wet (Fig. 16). Arrested dentin caries is hard to moderately soft; it is dark brown in color and has a dry appearance (Fig. 17). The tissue changes in the dentin vary markedly between acute and arrested caries. These differences are the main reasons which make them unsuitable for experimental studies, because the starting point for the subsequent evaluations will not be known. 
The demineralization of the enamel and dentin is the predominant characteristic of acute caries and it increases the permeability of the dentin. The mineral salts are lost into the oral environment and the porosities formed allow dyes to settle which promotes discoloration and becomes a characteristic clinical sign of caries. The progression of acute caries occurs at a rate which seems to overshadow any defense mechanisms that may take place in the dentin. Sensitivity to hot, cold and sweets are common early clinical symptoms and if left untreated severe pulp reactions will develop and then the odontoblasts subjacent to the affected dentin may be destroyed. Under favorable conditions, including appropriate restorative treatment or fracture of the overlaying enamel making the lesion self cleansing, new secondary odontoblasts may be differentiated from the cells in the pulp. Interface dentin will form followed by tertiary dentin. If these defense mechanisms do not come into play, acute pulpits will be manifested and the healing of the pulp will be compromised. Endodontic treatment or extraction may be the only alternative treatments.

Slowly progressing caries may end up as arrested caries lesions. Changes in the oral hygiene or fracture of the enamel over the superficial part of the lesion which make it self cleansing, may assist in changing the status of an acute lesion to an arrested lesion. The changes in both the dentin and in the pulp subjacent to the lesion are less dramatic than in quickly progressing acute caries.

Occlusion of the tubules by mineral deposits subjacent to slowly progressing caries (7) resulting

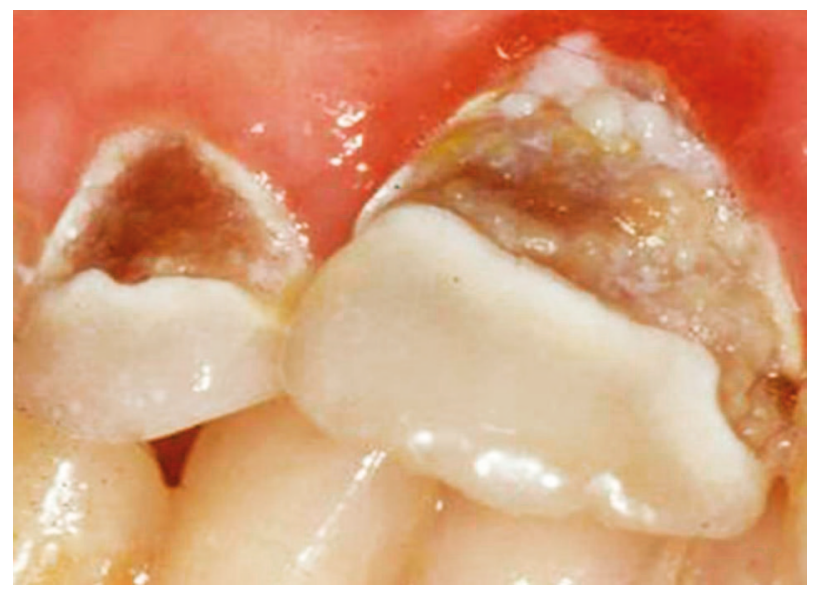

Figure 16. Clinical illustration of active caries lesion. The lesions are soft, slightly discolored, wet looking, and covered by plaque. in a 'transparent zone' subjacent to the demineralized carious dentin (Fig. 12a). This zone is hypermineralized (Fig. 12b) due to the mineral salts occluding the tubules. These deposits are crystalline in structure. Needle and rhombohedral shaped crystals have been identified as various forms of calcium phosphates, including hydroxyapatite and whitlockite crystals. Such intratubular crystal depositions are characteristic of slowly progressing and arrested dentin caries and of non-sensitive exposed dentin. The mineralized components of the dentin, including the crystals deposited in the tubules, are dissolved during the advancing caries. The most of these minerals are lost as the caries progresses, but if the progression is slow, some mineral salts are re-precipitated as intratubular crystals. This re-crystalinization results in the "transparent zone of caries' which can be observed as a highly mineralized area subjacent to the demineralized part of the lesion on microradiographs and sometimes on clinical radiographs. This type of occlusion of the tubules is a physicochemical type of defense mechanism that markedly reduces the permeability of the affected dentin.

\section{Bonding Materials to Dentin}

Recent attention to dentin permeability relates to the penetration of resin monomers into dentin. The penetration of resin monomer into dentin tubules and their branches (Figs. 15a-b) and its impregnation of the thin layer of demineralized intertubular collagen matrix exposed as a result of acid etching (Fig. 5d), are

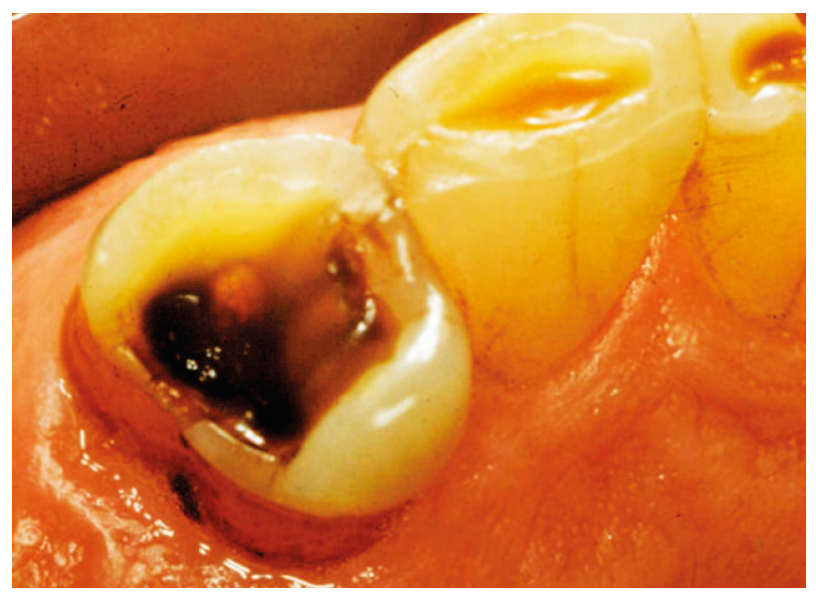

Figure 17. Clinical illustration of arrested caries. The dentin is hard, darkly discolored, dry looking and plaque free. 
essential components in bonding resin based restorations to dentin. When dealing with dentin in newly erupted teeth, the penetration into the tubules has to take place against a pressure gradient (Figs. 18a-b). The thin layer of collagen exposed as a result of the demineralization will be impregnated by resin to form a hybrid layer. It is important to realize that the washing of the surface with a water spray after acid etching will leave a dentin surface with no smear layer or smear plugs.

The relatively high tissue fluid pressure in the pulp will counteract the penetration of resin monomer into dentin, especially in newly erupted teeth with open tubules. The formation of resin tags will be facilitated in tubules that have no direct communication to the pulp. In situations where 'dead tracts' have formed, the high intratubular tissue fluid pressure is not present. Dead tracts in dentin are common whenever tertiary dentin has formed, e.g., as a reaction to active caries lesions, or in teeth from individuals where the tubules have become mineralized.

Some parts of the root dentin have few tubules and some small areas are devoid of tubules (8). Obturation of the root canal as the final stage in endodontic treatment using adhesive techniques must therefore depend on the

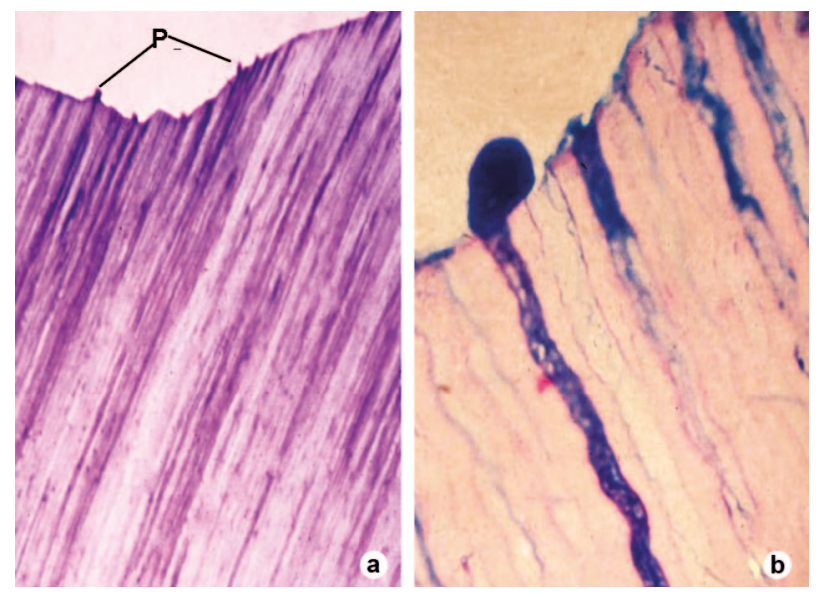

Figure 18. Images from toluidine stained section of a demineralized newly erupted tooth extracted immediately after a cavity preparation performed under an abundant water spray. Note the protrusions $(\mathrm{P})$ of the content from some of the tubules (Fig. 18a). The protrusion is not shown from all tubules presumably due to blockage of the opening of the tubules by the smear layer. Higher magnification of the protruded contents is shown in Fig. $18 \mathrm{~b}$ from a section of a tooth extracted immediately after cavity preparation similar to that in Fig. 18a. impregnation of exposed collagen forming a hybrid layer but this is clinically difficult to achieve.

Acidic cements applied directly to prepared dentin following cavity or crown preparation will dissolve the smear layer, but since the dentin debris is trapped by the cement, the smear layer becomes part of the hybrid layer that is likely to prevent penetration of the noxious acid to the pulp. This reduction in permeability has not been demonstrated experimentally, but extensive clinical experience over more than a century using zinc phosphate cements to secure inlays and crowns in place has been successful. The use of self-etching adhesive materials work in much the same way since the demineralized components will be trapped by the material. The limited demineralization of the prepared surface by acidic materials will restrict the formation of a classical hybrid layer and also reduce the possibilities for resin tag formation.

\section{Final Considerations}

Tubules extending through the dentin are the pathway for noxious agents towards the pulp and for transmission of sensitive stimuli. They also allow penetration of resin monomer which may be polymerized and become important in adhesive dentistry.

Highly mineralized peritubular dentin lines the tubules and their branches. They are formed during dentinogenesis of the main part of coronal dentin. Two basically different mechanisms for occlusion of dentinal tubules can be recognized. One mechanism involves the gradual growth of the peritubular dentin along the entire length of the tubules and an intratubular mineralization which may take place in the tubules in the most pulpal part of the dentin. These types of obturation of the tubules are under the control of odontoblasts. The other mechanism for occlusion of dentinal tubules is a physicochemical precipitation of mineral deposits within the tubules which is not under the control of odontoblasts. Irrespective of the mechanism for occlusion or obturation of dentinal tubules by mineralized deposits, the result will be reduced permeability of the dentin and it may become impermeable.

A number of clinical conditions will affect the structure of dentin and its permeability. Apart from the increased intratubular mineralization, formation of tertiary dentin at the pulpal aspect of affected dentin may take place. Both of these processes are essential 
biological reactions which should be taken into consideration in treating teeth. They represent important defense mechanism to noxious agents by affecting the permeability of the dentin. The first formed tertiary dentin may be an atubular 'interface' dentin which acts as an impermeable barrier between the primary-secondary dentin continuum and the tertiary dentin.

Dental caries, especially slowly progressing caries, show occlusion of tubules by re-precipitation of some of the dissolved mineral salts within the tubules. In addition the formation of tertiary dentin occurs at the pulpal aspect of the tubules affected by caries. Both the occlusion of the tubules and the tertiary dentin formation will reduce the permeability of the dentin.

\section{RESUMO}

A permeabilidade envolve a passagem de fluidos, íons, moléculas, material particulado e bactérias para o interior e através de uma substância ou tecido sob diferentes e variáveis condições. A permeabilidade da dentina é essencial para a fisiologia e para os padrões de reação do órgão dentino-pulpar. Nutrientes e impulsos são transportados a partir da polpa por meio dos processos odontoblásticos e o conteúdo dos seus túbulos mantém a vitalidade da dentina. Entretanto, o principal interesse deste artigo concentrase na penetração do exterior para a polpa e não da polpa para o exterior. O presente artigo centra-se essencialmente nos túbulos dentinários; como eles são formados e como eles mudam em razão da função normal e anormal, idade e processos patológicos, e o efeito destes processos na permeabilidade da dentina. Especial atenção é dada à patência dos túbulos dentinários. Este artigo apresenta uma visão geral do assunto e baseia-se amplamente nas pesquisas e percepções clinicas do próprio autor e em sua ativa participação na educação odontológica continuada ao longo dos últimos 50 anos. Não se trata de uma revisão da literatura relacionada à permeabilidade dentinária, mas sim da interpretação de resultados relacionados à permeabilidade dentinária com base na experiência e opiniões formadas ao longo de toda uma vida dedicada à pesquisa odontológica.

\section{REFERENCES}

1. Mjör IA, Nordahl I. The density and branching of dentinal tubules in human teeth. Archs Oral Biol 1996;41:401-412.

2. Pashley DH, Pashley EL. Dentin permeability and restorative dentistry: a status report for the Amer J Dent 1991;4:5-9.

3. Mjör IA. Dentinets permeabilitet. Odontol Tid 1969;77:257-276.

4. Mjör IA. Pulp-dentin biology in restorative dentistry. Chicago: Quintessence; 2002.

5. Fish EW. An Experimental Investigation of Enamel and Dentin and the Dental Pulp. London: John Bale, Sons \& Danielson, Ltd.

6. Senawongse P, Otsuki M, Tagami M, Mjör IA. Morphological characterization of attrited human dentine. Arch Oral Biol 2008;53:14-19.

7. Frank M, Wolff F, Gutmann B. Micoscopie electonique de la carie au niveau de la dentine humaine. Archs Oral Biol 1964;9:163-179.

8. Mjör IA, Smith MR, Ferrari M, Mannocci F. The structure of dentine in the apical region of human teeth. Int Endod J 2001;34:346353.

9. Barber D, Massler M. Permeability of active and arrested carious lesions to dyes and radioactive isotopes. J Dent Child 1964;31:2633.

10. Yoshiyama M, Masada J, Uchida A, Ishida, H. Scanning electron microscopic characterization of sensitive vs. insensitive human radicular dentin. J Dent Res 1989;68:1498-1502.

11. Bergman G. Studies on mineralized tissues. XIII - Combined microradiographic and autoradiographic investigations on carious teeth. J Dent Belge 1959:59:75-86.

12. Takuma S. Ultrastructure of dentinogenesis. In: Miles EAW (Editor.). Vol. 1: Structural and Chemical Organization of Teeth. New York: Academic Press 1967:355-370. 